BMJ

Open

Gastroenterology

\title{
Gastrointestinal mucosal damage in patients with COVID-19 undergoing endoscopy: an international multicentre study
}

Giuseppe Vanella (D) , ${ }^{1}$ Gabriele Capurso, ${ }^{1}$ Cesare Burti, ${ }^{2}$ Lorella Fanti, ${ }^{3}$ Luigi Ricciardiello, ${ }^{4}$ Andre Souza Lino, ${ }^{5}$ Ivo Boskoski, ${ }^{6,7}$ Michiel Bronswijk, ${ }^{8}$ Amy Tyberg, ${ }^{9}$ Govind Krishna Kumar Nair, ${ }^{10}$ Stefano Angeletti, ${ }^{11}$ Aurelio Mauro, ${ }^{12}$ Fabiana Zingone, ${ }^{13}$ Kofi W. Oppong, ${ }^{14}$ Daniel de la Iglesia-Garcia, ${ }^{15}$ Lieven Pouillon, ${ }^{16}$ loannis S. Papanikolaou, ${ }^{17}$ Pierluigi Fracasso, ${ }^{18}$ Fabio Ciceri, ${ }^{19}$ Patrizia Rovere-Querini, ${ }^{20}$ Carolina Tomba, ${ }^{2}$ Edi Viale, ${ }^{3}$ Leonardo Henry Eusebi, ${ }^{4}$ Maria Elena Riccioni, ${ }^{6,7}$ Schalk van der Merwe, ${ }^{8,21}$ Haroon Shahid, ${ }^{9}$ Avik Sarkar, ${ }^{9}$ Jin Woo (Gene) Yoo, ${ }^{10}$ Emanuele Dilaghi, ${ }^{11}$ R. Alexander Speight, ${ }^{14}$ Francesco Azzolini, ${ }^{3}$ Francesco Buttitta, ${ }^{4}$ Serena Porcari, ${ }^{6,7}$ Maria Chiara Petrone, ${ }^{1}$ Julio Iglesias-Garcia, ${ }^{15}$ Edoardo V. Savarino, ${ }^{13}$ Antonio Di Sabatino, ${ }^{12}$ Emilio Di Giulio, ${ }^{11}$ James J. Farrell, ${ }^{10}$ Michel Kahaleh, ${ }^{9}$ Philip Roelandt, ${ }^{8,21}$ Guido Costamagna, ${ }^{6,7}$ Everson Luiz de Almeida Artifon, ${ }^{5}$ Franco Bazzoli, ${ }^{4}$ Per Alberto Testoni, ${ }^{3}$ Salvatore Greco, ${ }^{2}$ Paolo Giorgio Arcidiacono ${ }^{1}$

To cite: Vanella G, Capurso G, Burti C, et al. Gastrointestinal mucosal damage in patients with COVID-19 undergoing endoscopy: an international multicentre study. BMJ Open Gastro 2021;8:e000578. doi:10.1136/ bmjgast-2020-000578

- Additional material is published online only. To view, please visit the journal online (http://dx.doi.org/10.1136/ bmjgast-2020-000578).

Received 23 November 2020 Revised 28 January 2021 Accepted 2 February 2021

Check for updates

(C) Author(s) (or their employer(s)) 2021. Re-use permitted under CC BY-NC. No commercial re-use. See rights and permissions. Published by BMJ.

For numbered affiliations see end of article.

Correspondence to Professor Paolo Giorgio Arcidiacono;

arcidiacono.paologiorgio@hsr.it

\section{ABSTRACT}

Background Although evidence suggests frequent gastrointestinal (Gl) involvement during coronavirus disease 2019 (COVID-19), endoscopic findings are scarcely reported. Aims We aimed at registering endoscopic abnormalities and potentially associated risk factors among patients with COVID-19.

Methods All consecutive patients with COVID-19 undergoing endoscopy in 16 institutions from highprevalence regions were enrolled. Mann-Whitney U, $\chi^{2}$ or Fisher's exact test were used to compare patients with major abnormalities to those with negative procedures, and multivariate logistic regression to identify independent predictors.

Results Between February and May 2020, during the first pandemic outbreak with severely restricted endoscopy activity, 114 endoscopies on 106 patients with COVID-19 were performed in 16 institutions (men $=70.8 \%$, median age $=68$ (58-74); 33\% admitted in intensive care unit; $44.4 \%$ reporting Gl symptoms). $66.7 \%$ endoscopies were urgent, mainly for overt Gl bleeding. 52 (45.6\%) patients had major abnormalities, whereas 13 bled from previous conditions. The most prevalent upper GI abnormalities were ulcers (25.3\%), erosive/ulcerative gastro-duodenopathy (16.1\%) and petechial/haemorrhagic gastropathy (9.2\%). Among lower Gl endoscopies, 33.3\% showed an ischaemic-like colitis.

Receiver operating curve analysis identified D-dimers $>1850 \mathrm{ng} / \mathrm{mL}$ as predicting major abnormalities. Only D-dimers $>1850 \mathrm{ng} / \mathrm{mL}(\mathrm{OR}=12.12(1.69-86.87))$ and presence of $\mathrm{Gl}$ symptoms $(\mathrm{OR}=6.17(1.13-33.67))$ were independently associated with major abnormalities at multivariate analysis.

\section{Summary box}

What is already known about this subject?

- Gastrointestinal (Gl) symptoms are found in more than $10 \%$ of patients with COVID- 19 .

- SARS-CoV-2 RNA is isolated in more than $40 \%$ of faecal samples of patients with COVID-19.

- The ACE-2 receptor is highly expressed in the Gl tract.

- Evidence on endoscopic findings in COVID-19 is limited to sporadic case report or series.

What are the new findings?

- In an international prospective endoscopy registry of patients with COVID-19 requiring urgent endoscopy, almost half were found to have an acute major $\mathrm{G}$ mucosal injury.

- In this highly selected population, erosive/ulcerative damage was the most prevalent endoscopic abnormality and an ischaemic-like colopathy was commonly (33.3\%) observed.

Conclusion In this highly selected cohort of hospitalised patients with COVID-19 requiring endoscopy, almost half showed acute mucosal injuries and more than one-third of lower Gl endoscopies had features of ischaemic colitis. Among the hospitalisation-related and patient-related variables evaluated in this study, D-dimers above 1850 $\mathrm{ng} / \mathrm{mL}$ was the most useful at predicting major mucosal abnormalities at endoscopy.

Trial registration number ClinicalTrial.gov (ID: NCT04318366). 


\section{Summary box}

How might it impact on clinical practice in the foreseeable future?

- Studies with greater inclusion of hospitalised and community-based patients with COVID-19 are needed to accurately determine the prevalence of gastrointestinal pathologies during active infection.

- More thorough clinical and translational investigation is needed to elucidate an eventual ischaemic aetiopathogenesis of COVID-19 gastrointestinal involvement, potentially carrying relevant therapeutic or preventive implications.

\section{INTRODUCTION}

Despite being primarily a respiratory disease, coronavirus disease 2019 (COVID-19), caused by the severe acute respiratory syndrome coronavirus 2 (SARS-CoV-2), can present with a wide spectrum of clinical manifestations and has been associated with extrapulmonary tropism and organ dysfunction ${ }^{1-3}$

Gastrointestinal (GI) symptoms have been recorded in more than $10 \%$ of affected patients, ${ }^{4-6}$ with only a fraction explainable by a systemic illness or the hospitalisation itself, while a quota of patients with COVID-19 presents GI symptoms without or before the onset of respiratory manifestations. ${ }^{6} 7$ This is not surprising, as the ACE-2 receptor, by which the SARS-CoV-2 invades cells, has been demonstrated to be abundantly expressed in the stomach, small intestine and rectum with a relatively higher intestinal expression with respect to other tissues. $^{6-10}$

Indeed, SARS-CoV-2 can been detected in faecal samples of more than $40 \%$ of patients with COVID-19, and a faecal-oral transmission has been hypothesised alongside the more common respiratory spread through droplets. $^{11} 12$ For these reasons, all major endoscopy scientific societies recommended high-level protection measures during upper and lower GI endoscopic procedures in patients who are COVID-19 positive or in areas with high disease prevalence. ${ }^{13-16}$

Evidence on endoscopic findings in patients with COVID-19 is limited to sporadic case report or series, ${ }^{17}$ describing non-specific findings, and suggesting that the virus directly damages GI mucosal surfaces. ${ }^{18}$

In addition, increasing evidence has been suggesting that vascular changes might be a distinctive feature of COVID- $19^{19}$ and that systemic endothelial damage and consequent disseminated microvascular thrombosis might act as a primer for many organs' damage, including the pulmonary one (the 'microvascular thromboinflammatory syndrome' theory). ${ }^{120}$ It is therefore tempting to hypothesise that such ischaemic damage might also occur in the GI tract.

The present multicentre study was therefore aimed at (1) systematically describing GI findings during endoscopic examination of patients with SARS-CoV-2 infection and (2) investigating factors associated with GI injury to gain more knowledge on its pathogenesis.

\section{METHODS}

\section{Study design and criteria for inclusion}

We planned a multicentre cross-sectional study to record endoscopic findings among all consecutive patients who are SARS-CoV-2 positive undergoing any GI endoscopic examination in 16 centres in high-incidence areas (online supplemental table 1 for centres and relative case contributions) during the pandemic phase (February - May 2020). In this phase, representing the first pandemic wave, access to endoscopy exams was severely restricted, and elective procedures were postponed. ${ }^{13-15}$

Endoscopic examinations were includible if performed on an adult patient with confirmed SARS-CoV-2 infection, ${ }^{21}$ before an eventual negative test indicating recovery with viral elimination (online supplemental statement 1 for full inclusion criteria).

\section{Variables and outcomes}

Trained investigators at each participating centre completed a case report form (CRF; online supplemental figure 1) assessing several patient-related and hospitalisation-related variables (online supplemental statement 2) relevant in the COVID-19 scenario. ${ }^{22-24}$ Overall mortality was registered.

Endoscopy findings were recorded according with the Minimal Standard Terminology (MST) for gastrointestinal endoscopy published by the World Endoscopy Organization $^{25}$ (online supplemental figure 1); histopathology was recorded when performed as clinically indicated.

All possible abnormalities as identified by the MST were a priori categorised as: chronic/acute-on-chronic (AoC) / minor/major as detailed in online supplemental table 2. AoC abnormalities mainly included bleeding from known chronic diseases, such as oesophageal varices in patients with cirrhosis, and were excluded from the risk factors analysis due to the chronic nature and the specific background fragility.

CRFs data extraction and categorisation of endoscopic findings were blindly assessed by two experienced endoscopists, with disagreements resolved by the opinion of a third one.

Consistent with the extensive classification according to MST, endoscopists performing the procedure were asked to indicate a final interpretation of endoscopic abnormalities based on patterns and distribution ${ }^{26}$ (local assessment). Extensive findings, local interpretation and histology (when available) were used for deciding an overall diagnosis at the moment of data extraction (central assessment). Multiple findings were allowed per each examination. The 'most severe' finding was used to categorise each single examination according to the aforementioned criteria.

The study protocol conforms to the Declaration of Helsinki and informed consent was obtained from each patient. 


\section{Statistical analysis}

Data are reported as frequency (proportions) for categorical variables. Data are reported as median (IQR) for continuous variables after normal distribution was excluded by the D'Agostino-Pearson test.

A receiver operating curve (ROC) analysis was performed for D-dimer values and platelet counts to identify the best threshold able to discriminate between patients with major abnormalities and patients with minor, chronic or no abnormalities ('negative' endoscopies). Area under the curve (AUC) and $\mathrm{p}$ value are reported for the ROC analysis together with sensitivity, specificity, positive and negative likelihood ratios (+LRand $-\mathrm{LR})$ for the identified cut-off.

Patients with major abnormalities detected were compared with patients with 'negative' endoscopies. A Mann-Whitney $\mathrm{U}$ test was used for continuous variables. A $\chi^{2}$ test was used for categorical variables, except in cases where total number of observations was $<20$ or at least one expected value was $<5$ and a Fisher's exact test was adopted. Variables demonstrating significant differences at univariate analysis were included in a multivariate logistic regression model, using an 'enter' method, with a p-to-enter $<0.05$ and a p-to-stay $<0.1$. Categorisation of variables is reported in online supplemental statement 3. Results are expressed as OR and 95\% CI, adjusted for age, sex and pre-admission ASA (AmericanSociety of Anesthesiologists) score. $\mathrm{P}<0.05$ (two-tailed) was considered statistically significant. Due to the exploratory nature of the study, no correction for multiple testing was performed.

MedCalc Statistical Software (Ostend, Belgium) was used for statistical analysis.

\section{RESULTS}

\section{Patients}

In 16 centres (based in Italy, Belgium, Spain, Greece, UK, Brasil and USA), admitting almost 17.000 patients with SARS-CoV-2, 114 endoscopies were performed between $20^{\text {th }}$ February 2020 and $8^{\text {th }}$ May 2020 on 106 patients.

Patients features are detailed in table 1; $75(70.8 \%)$ were men, median age was $68(58-74)$ years; the most frequent comorbidities were hypertension $(52.4 \%)$ and diabetes $(21.9 \%)$.

Sixty-seven per cent of the patients were admitted in non-intensive care unit settings (NIU) and 33\% were in intensive care unit (ICU) for invasive ventilation management. No patient in this cohort was managed as outpatient.

After excluding 16 patients admitted with other underlying symptomatic abdominal diseases (eg, acute pancreatitis, cholecystitis), $55.6 \%$ of the remaining patients were free of GI symptoms, while the prevalence of abdominal pain, nausea, diarrhoea, vomiting and anorexia was respectively $27.8 \%, 16 \%, 15.6 \%, 14.4 \%$ and $11.1 \%$ (table 1).
Table 1 Demographics and clinical features of the study population

\begin{tabular}{ll}
\hline Characteristic & $\mathbf{n = 1 0 6}$ \\
\hline Male, $\mathrm{n}(\%)$ & $75(70.8 \%)$ \\
\hline Age, years (IQR) & $68(58-74)$ \\
\hline Ethnicity & \\
\hline Non-Hispanic White & $102(96.2 \%)$ \\
\hline Hispanic & $2(1.9 \%)$ \\
\hline Black/African-American & $2(1.9 \%)$ \\
\hline Pre-admission ASA score & \\
\hline 1 & $11(10.4 \%)$ \\
2 & $38(35.8 \%)$ \\
3 & $52(49.1 \%)$ \\
\hline 4 & $5(4.7 \%)$ \\
\hline
\end{tabular}

\section{Comorbidities}

\begin{tabular}{|c|c|}
\hline Hypertension & $55 / 105(52.4 \%)$ \\
\hline Diabetes & $23 / 105(21.9 \%)$ \\
\hline Ischaemic cardiomyopathy & $17 / 105(16.2 \%)$ \\
\hline CKD & $15 / 105(14.3 \%)$ \\
\hline Obesity & $12 / 105(11.4 \%)$ \\
\hline COPD & $11 / 105(10.5 \%)$ \\
\hline Atrial fibrillation & $10 / 105(9.5 \%)$ \\
\hline Active cancer & 9/105 (8.6\%) \\
\hline Cirrhosis & 9/105 (8.6\%) \\
\hline \multicolumn{2}{|l|}{ Reason for admission } \\
\hline COVID-19 related & $72(67.9 \%)$ \\
\hline Other (incidental SARS-CoV-2 diagnosis) & $34(32.1 \%)$ \\
\hline COVID respiratory disease & $86(81.1 \%)$ \\
\hline Gl symptoms & $\mathrm{n}=90^{*}$ \\
\hline None & $50(55.6 \%)$ \\
\hline At least one symptom $†$ & $40(44.4 \%)$ \\
\hline Abdominal pain & $25(27.8 \%)$ \\
\hline Nausea & $16(17.8 \%)$ \\
\hline Diarrhoea & $14(15.6 \%)$ \\
\hline Vomiting & $13(14.4 \%)$ \\
\hline Anorexia & $10(11.1 \%)$ \\
\hline \multicolumn{2}{|l|}{ Hospital regimen } \\
\hline Intensive care unit, with invasive ventilation & $35(33 \%)$ \\
\hline Subintensive care & $71(67 \%)$ \\
\hline \multicolumn{2}{|l|}{ Relevant therapy during admission } \\
\hline Antibiotics/antimicotic & 90/101 (89.1\%) \\
\hline Anticoagulation & 46/84 (54.8\%) \\
\hline Antivirals & 45/98 (45.9\%) \\
\hline Hydroxychloroquine & $41 / 99(41.4 \%)$ \\
\hline Biological therapy & 15/97 (15.5\%) \\
\hline Steroids & $28 / 100(28 \%)$ \\
\hline
\end{tabular}

*Excluding patients with symptoms related to other known abdominal diseases.

†More than one symptom admittible.

ASA, American Society of Anesthesiologists; CKD, chronic kidney disease; COPD, chronic obstructive pulmonary disease; Gl, gastrointestinal. 


\section{Endoscopic examinations}

Reasons for performing the endoscopic procedures are detailed in online supplemental table 3, with $66.7 \%$ of exams being performed in an urgent setting, most frequently (55.3\%) due to overt GI bleeding.

According to the aforementioned classification of the endoscopic findings, $52(45.6 \%)$ patients were diagnosed with major abnormalities, $13(11.4 \%)$ of patients with an
AoC abnormality, while $12.3 \%, 3.5 \%$ and $27.2 \%$ were diagnosed with minor, chronic or no abnormality, respectively (online supplemental table 3 ).

Detailed endoscopic findings are reported in table 2.

Among upper GI (UGI) endoscopies, the most prevalent abnormalities were ulcers $(25.3 \%$; of which $50 \%$ actively bleeding and $13.6 \%$ with signs of recent bleeding; see figure 1), erosive/ulcerative diffuse damage (16.1\%)

Table 2 Prevalence of endoscopic abnormalities. Multiple abnormalities admitted for each examination

\begin{tabular}{|c|c|c|c|}
\hline Upper GI examinations & $\mathrm{n}=87$ & Lower GI examinations & $\mathrm{n}=\mathbf{2 7}$ \\
\hline Normal examinations, $\mathrm{n}(\%)$ & $26(29.9 \%)$ & Normal examinations, $\mathrm{n}(\%)$ & $5(18.5 \%)$ \\
\hline Major findings & & Major findings & \\
\hline Oesophagitis & $7(8 \%)$ & Ischaemic-like colopathy & 9 (33.3\%) \\
\hline Los Angeles A/B oesophagitis & 4 & Diffuse & 3 \\
\hline Los Angeles C/D oesophagitis & 3 & Left colon & 6 \\
\hline Petechial/haemorrhagic gastropathy & $8(9.2 \%)$ & Lower bleeding w/o other abnormalities & $3(11.1 \%)$ \\
\hline Erosive/ulcerative diffuse damage & $14(16.1 \%)$ & Minor findings & \\
\hline Gastric & 8 & Unspecific left colon erythema & $2(7.4 \%)$ \\
\hline Duodenal & 3 & Chronic findings & \\
\hline Antro-duodenal & 2 & Segmental colitis associated with diverticulosis & $3(11.1 \%)$ \\
\hline Jejunal & 1 & Diverticulosis & $4(14.8 \%)$ \\
\hline Ulcers & $22(25.3 \%)$ & Haemorrhoids & $1(3.7 \%)$ \\
\hline Gastric & $11(12.6 \%)$ & Acute-on-chronic findings & \\
\hline Forrest III (non-bleeding) & 6 & Ulcerative inflammatory colitis & $1(3.7 \%)$ \\
\hline Forrest Ilb (visible clot) & 1 & Bleeding diverticulum & $4(14.8 \%)$ \\
\hline Forrest la/b (oozing/spurting) & 4 & Bleeding angioectasia & $2(7.4 \%)$ \\
\hline Duodenal & $9(10.3 \%)$ & & \\
\hline Forrest llb (visible clot) & 2 & & \\
\hline Forrest la/b (oozing/spurting) & 7 & & \\
\hline Oesophageal (non-bleeding) & 2 & & \\
\hline Dieulafoy lesion & $1(1.1 \%)$ & & \\
\hline \multicolumn{4}{|l|}{ Minor findings } \\
\hline Erythematous/oedematous gastropathy & $21(24.1 \%)$ & & \\
\hline Erythematous/oedematous duodenopathy & $6(6.9 \%)$ & & \\
\hline Granular/nodular gastropathy & $2(2.3 \%)$ & & \\
\hline Oesophageal candidiasis & $2(2.3 \%)$ & & \\
\hline \multicolumn{4}{|l|}{ Chronic } \\
\hline Gastric antral vascular ectasia & $3(3.5 \%)$ & & \\
\hline Atrophic gastropathy & $3(3.5 \%)$ & & \\
\hline Oesophageal varices & $5(5.9 \%)$ & & \\
\hline Cystic glands polyps & $1(1.2 \%)$ & & \\
\hline Angioectasia & $1(1.2 \%)$ & & \\
\hline \multicolumn{4}{|l|}{ Acute-on-chronic } \\
\hline Oozing oesophageal varices & $3(3.4 \%)$ & & \\
\hline Oozing gastric polyp & $2(2.3 \%)$ & & \\
\hline Oozing hypertensive duodenopathy & $1(1.2 \%)$ & & \\
\hline Oozing GAVE & $1(1.2 \%)$ & & \\
\hline
\end{tabular}

GAVE, gastric antral vascular ectasia; GI, gastrointestinal; w/o, without. 


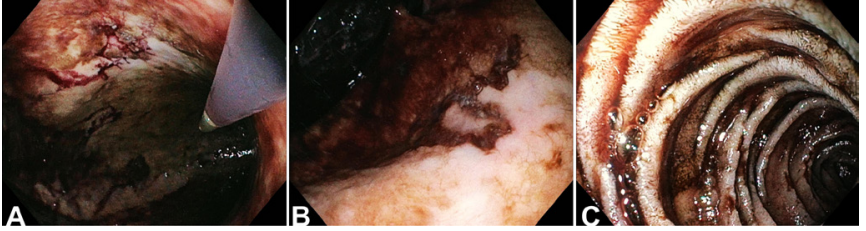

Figure 1 Upper Gl tract. A patient admitted in intensive care unit underwent upper Gl endoscopy on day 15 after admission for haematemesis. Digested blood was found in the stomach and duodenum, together with a superficial ulcer with irregular margins with flat pigmentation (Ilc according to Forrest classification) in the lesser curvature, on a pale and dystrophic background mucosa $(A, B)$. In the second duodenal portion, small ulcers were found on an atrophic background mucosa (C). Histology of the ulcer showed ischaemic damage with sporadic endocapillary microthrombi. Gl,gastrointestinal.

and petechial/haemorrhagic gastropathy (9.2\%); see figure 2. Of note, one case consistent with acute oesophageal necrosis or 'black oesophagus' associated with a haemorrhagic gastropathy was also recorded.

Only one enteroscopy was performed: a 75 years old patient without medical history or chronic therapy underwent a CT scan for persistent abdominal pain, showing thickened jejunal walls; enteroscopy showed multiple erosions and ulcers (see figure 3), whose histology reported acute inflammation, ulcerations and granulation tissue, with abundant eosinophils and shortening of villi, without definite aetiology (all cultural exams negative including immunohistochemistry for Cytomegalovirus and PCR for SARS-CoV-2).

Among lower GI (LGI) endoscopies, 33.3\% were found to have an ischaemic-like colonic damage (see figure 4), in one case graded as severe due to colonic necrosis (see figure 5).

The rate of major findings was similar between lower and upper GI tract endoscopies, while 'negative' examinations were less prevalent in the lower tract, where AoC abnormalities were reported more frequently $(p=0.02$ (online supplemental table 4)).

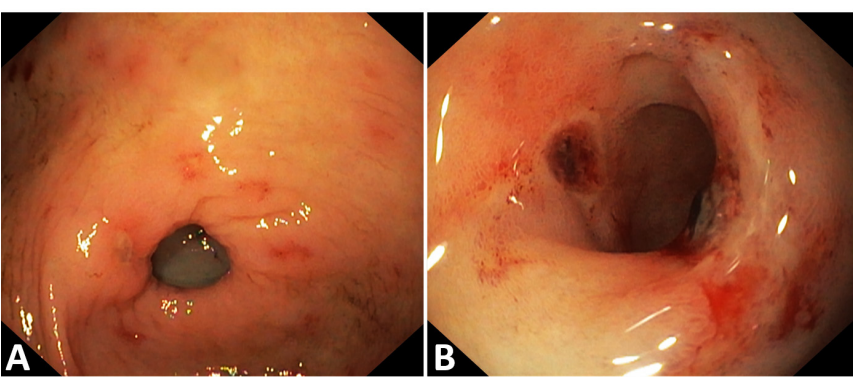

Figure 2 Gastroduodenal damage in a patient undergoing upper GI endoscopy for melena after being admitted for COVID-19. Gastroscopy showed diffuse erythema, antral erosions and one non-bleeding prepyloric ulcer (A). In the bulb a dystrophic mucosa showed diffuse congestion, petechiae (submucosal haemorrhages) and a blackish appearance of some disepithelialised areas (B). Gl,gastrointestinal

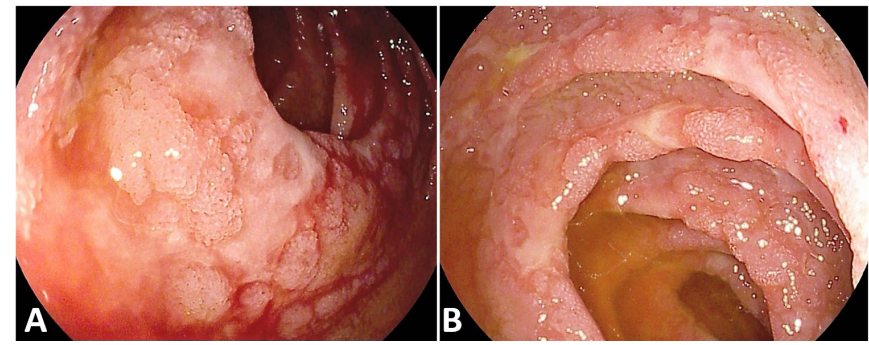

Figure 3 Small intestine. A patient without medical history or chronic therapy, admitted in a non-intensive ward, underwent a CT scan for persistent abdominal pain, showing thickened jejunal walls; enteroscopy performed 44 days after admission showed multiple erosions and ulcers on a background atrophic mucosa with shortened villi. Histology reported acute inflammation, ulcerations and granulation tissue, with abundant eosinophils, without definite aetiology (all cultural exams negative including immunohistochemistry for Cytomegalovirus and PCR for SARS-CoV-2).

Time to endoscopy from COVID-19 onset and hospital admission are reported in online supplemental table 4; while there was no significant difference in the prevalence of findings according to admission-to-endoscopy time, examinations executed within 7 days from COVID-19 onset had a $67.9 \%$ prevalence of major findings whereas this rate was $45.2 \%$ in those executed afterwards $(p=0.04)$.

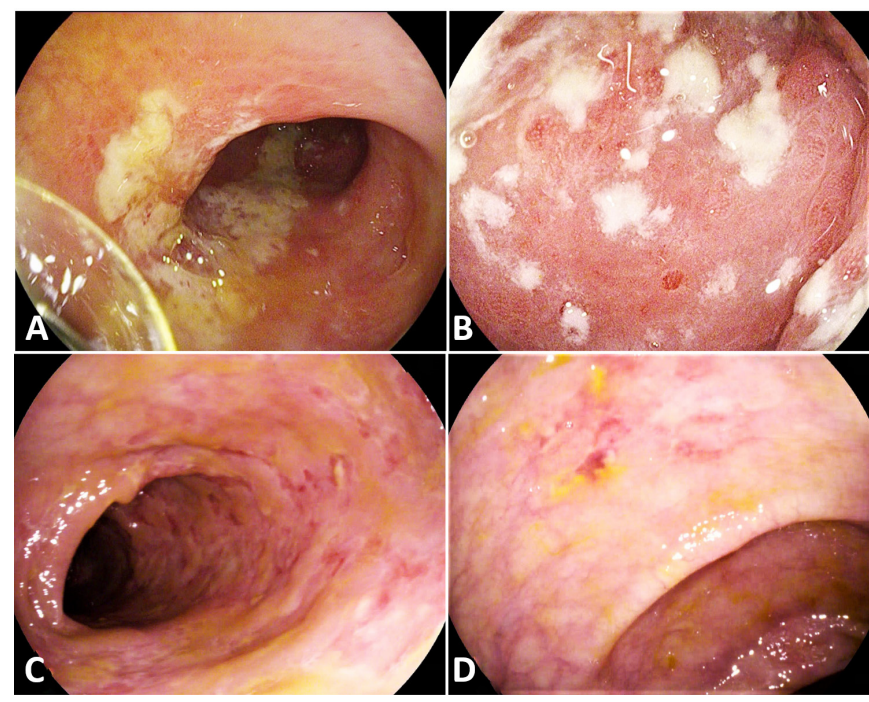

Figure 4 Colon. Endoscopy appearance of ischaemiclike colopathy. $(A, B)$ An elderly patient was admitted with diarrhoea and COVID-19 pneumonia in a non-intensive ward. Five days after admission he underwent a colonoscopy showing friable mucosa with ulcers and pseudomembranes in the sigmoid and descending colon (B); the pathognomonic 'single stripe' sign (a linear ulcer extending longitudinally along the antimesenteric bowel wall) was visible in the sigmoid (A). Histology was compatible with ischaemic damage. $(C, D) A$ patient without relevant comorbidities or chronic therapy, underwent a colonoscopy for bloody diarrhoea 30 days after intensive care unit admission. Colonoscopy found petechiae, oedema and easily friable mucosa (C) with small erosions (D) with a patchy and segmental distribution in the left 'watershed' area (sigmoid). 


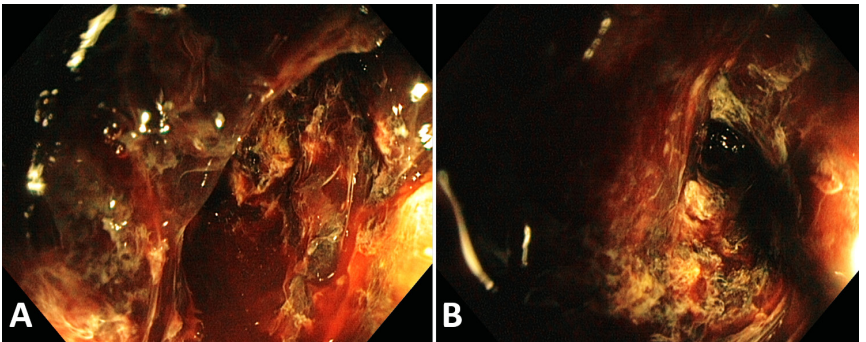

Figure 5 An elderly hospitalised patient with COVID-19 with diabetes, kidney failure and atrial fibrillation underwent urgent proctosigmoidoscopy for rectal bleeding. Endoscopy was interrupted due to the presence of abundant coagulated blood. Rinsing of clots showed a fragile, dusky, cyanotic and black mucosa with severe ulcerations, compatible with severe ischaemic/necrotic colitis.

\section{Histopathology}

Samples for histology were obtained in 12 patients $(10.5 \%)$.

Of the five biopsies on gastric ulcers, one was found to be gastric adenocarcinoma, two erosive chronic inflammation, one Helicobacter pylori-related active gastritis and one ischaemic damage with sporadic endocapillary microthrombi (see figure 1).

Of the six biopsies on erosive/ulcerated colonic mucosa, one was found with an active phase of a known inflammatory bowel disease (ulcerative colitis), while of the remaining five, four were found with aspects of coagulative necrosis, microhaemorrhages, microthrombi and vascular congestion, suggesting an ischaemic colitis and two with an erosed epithelium with a non-specific increase in inflammatory infiltrate (with endoscopy suggesting an ischaemic-like damage). In one case with histology consistent with ischaemic colitis, COVID-19 could be identified at immunohistochemistry (see figure 6). Of note, among the histologically confirmed cases of ischaemic colitis, were a 45-year-old woman and a 77-year-old man without previous known comorbidities (ASA 1) or chronic therapy.

\section{Association between clinical variables and endoscopic findings}

ROC curve analysis identified a D-dimers value above 1850 $\mathrm{ng} / \mathrm{mL}$ as the best discriminating thresholds to predict a higher probability of detection of a major abnormality (AUC $=0.6, \quad \mathrm{p}=0.08$; sensitivity $=51.3 \%$; specificity $=77.4 \%$; $+\mathrm{LR}=2.3$, $-\mathrm{LR}=0.6$; online supplemental figure 2). ROC curve analysis for platelet count did not find a sufficiently accurate threshold (AUC=0.5, $\mathrm{p}=0.5$ ) and therefore we continued to report this variable as continuous.

Comparing procedures showing major abnormalities to 'negative' examinations (table 3), there was a higher prevalence of male sex $(80.8 \%$ vs $65.3 \%, \mathrm{p}=0.08)$. No difference was noticed for pre-admission ASA score. Prevalence of atrial fibrillation was significantly lower among patients with major abnormalities $(3.8 \%$ vs $16.7 \%, \mathrm{p}=0.05)$. No difference was found for chronic non-steroidal anti-inflammatory drugs use, even when focusing on UGI endoscopies only, as well as

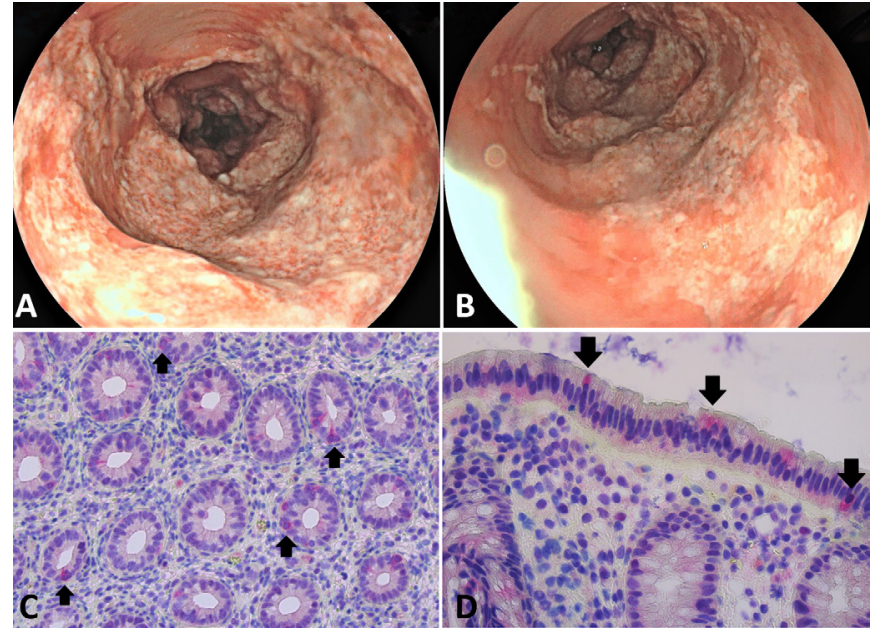

Figure 6 A middle-age patient, with a history of dyslipidaemia, was admitted following an episode of abdominal discomfort, rectal blood loss and a vasovagal syncope. On admission COVID-19-screening came back positive. Subsequent flexible sigmoidoscopy revealed unilateral ulcerations in the left colon with rectal sparing (A, B). Histopathology showed patchy atrophic changes, mucus depletion, signs of regeneration and infiltration by polymorphonuclear neutrophils, typical for an ischaemia-type colitis. COVID-19-antibody-staining was positive on various enterocytes (C, D arrows).

in the prevalence of Helicobacter pylori in the available histological samples $(1 / 5$ vs $1 / 4$ respectively).

D-dimers $>1850 \mathrm{ng} / \mathrm{mL}$ DDU were found in $48.6 \%$ of patients with major abnormalities versus $22.6 \%$ of 'negative' procedures $(\mathrm{p}=0.03)$.

Patients with major abnormalities reported more frequently at least one GI symptom $(53.1 \%$ vs $30 \%, \mathrm{p}=0.03$, especially abdominal pain $(\mathrm{p}=0.07)$ and diarrhoea $(\mathrm{p}=0.13))$.

No difference was detected in the rate of patients with or without COVID-19 respiratory involvement or admitted in the ICU versus NIU. A higher rate of biological therapy ( $22.9 \%$ vs $7 \%, \mathrm{p}=0.04$, mainly tocilizumab and anakinra) and antiviral $(56.2 \%$ vs $34.1 \%, \mathrm{p}=0.03$; mainly INN-lopinavir/ ritonavir and remdesivir) during admission was recorded among patients with major findings.

At univariate analysis atrial fibrillation $(\mathrm{OR}=0.2$ (0.040.99)), a D-dimer $>1850 \mathrm{ng} / \mathrm{mL}$ DDU (OR=3.25 (1.139.38)), presence of GI symptoms $(\mathrm{OR}=2.64(1.10-6.35))$, antiviral therapy $(\mathrm{OR}=2.49(1.07-5.79))$ and biological therapy $(\mathrm{OR}=3.96(1.03-15.33))$ were associated with the risk of a major finding.

Comparisons of procedures showing major versus AoC findings are shown in online supplemental table 5 .

\section{Multivariate logistic regression}

At multivariate analysis (see online supplemental table 6), D-dimers value $>1850 \mathrm{ng} / \mathrm{mL}$ DDU (OR=12.12 (1.69$86.87)$ ) and presence of at least one GI symptom $(\mathrm{OR}=6.17$ (1.13-33.67)) were independently associated with major endoscopic abnormalities, in a model adjusted for age, sex and pre-admission ASA score. 
Table 3 Patients-related and hospitalisation-related factors associated with major findings. In the columns on the left, prevalence of each variable is compared between procedures finding a major abnormality versus 'negative' procedures. In the rightmost column, univariate analysis is provided as OR and $95 \% \mathrm{Cl}$

\begin{tabular}{|c|c|c|c|c|}
\hline Characteristics & $\begin{array}{l}\text { Major abnormalities } \\
\mathrm{n}=52\end{array}$ & 'Negative' procedures $n=49$ & $P$ value & $\begin{array}{l}\text { Univariate analysis } \\
\text { OR }(95 \% \mathrm{Cl})\end{array}$ \\
\hline Male sex, n (\%) & $42(80.8 \%)$ & $32(65.3 \%)$ & 0.08 & \\
\hline Median age, years (IQR) & $71(62.5-79)$ & $68(57.5-72.3)$ & 0.11 & \\
\hline Pre-admission ASA score, $\mathrm{n}(\%)$ & & & 0.99 & \\
\hline ASA 1 & $6(11.5 \%)$ & $5(10.2 \%)$ & & \\
\hline ASA 2 & $20(38.5 \%)$ & $19(38.8 \%)$ & & \\
\hline ASA 3 & $24(46.2 \%)$ & $23(46.9 \%)$ & & \\
\hline ASA 4 & $2(3.8 \%)$ & $2(4.1 \%)$ & & \\
\hline \multicolumn{5}{|l|}{ Comorbidities } \\
\hline Hypertension, n (\%) & $30(57.7 \%)$ & $23(47.9 \%)$ & 0.33 & \\
\hline Diabetes, n (\%) & $8(15.4 \%)$ & $12(25 \%)$ & 0.23 & \\
\hline Ischaemic cardiomyopathy, n (\%) & $7(13.5 \%)$ & $8(16.7 \%)$ & 0.65 & \\
\hline Atrial fibrillation, n (\%) & $2(3.8 \%)$ & $8(16.7 \%)$ & 0.05 & 0.2 (0.04 to 0.99$)$ \\
\hline Active cancer, n (\%) & $3(5.8 \%)$ & $7(14.6 \%)$ & 0.19 & \\
\hline Cirrhosis & $2(3.9 \%)$ & $3(6.2 \%)$ & 0.67 & \\
\hline CKD & $10(19.2 \%)$ & $6(12.5 \%)$ & 0.36 & \\
\hline COPD/asthma & $7(13.5 \%)$ & $5(10.4 \%)$ & 0.76 & \\
\hline Obesity & $7(13.5 \%)$ & $5(10.4 \%)$ & 0.76 & \\
\hline Antiplatelet at admission & $17(32.7 \%)$ & $11(22.9 \%)$ & 0.38 & \\
\hline NSAIDs at admission & $16(30.8 \%)$ & $9(18.8 \%)$ & 0.17 & \\
\hline UGI endoscopies only & $12 / 37(32.4 \%)$ & $4 / 27(14.8 \%)$ & 0.15 & \\
\hline Anticoagulant at admission & $5(9.6 \%)$ & $11(22.9 \%)$ & 0.10 & \\
\hline Median D-dimer, ng/mL DDU $(\mathrm{IQR})^{*}$ & 2149 (567.8-3522.5) & $780(508.3-1762.5)$ & 0.09 & \\
\hline D-dimer $>1850 \mathrm{ng} / \mathrm{mL} \mathrm{DDU}^{*}$ & $18 / 37(48.6 \%)$ & $7 / 31(22.6 \%)$ & 0.03 & 3.25 (1.13 to 9.38$)$ \\
\hline Median platelet count, $n \times 10^{9} / \mathrm{L}(\mathrm{IQR})^{\star}$ & $231(177.5-304.8)$ & $275.5(148.5-349.5)$ & 0.52 & \\
\hline Symptoms, n (\%)* & $\mathrm{n}=49$ & $\mathrm{n}=40$ & & \\
\hline Any Gl symptom & $26(53.1 \%)$ & $12(30 \%)$ & 0.03 & 2.64 (1.10 to 6.35$)$ \\
\hline Nausea & $9(18.4 \%)$ & $5(12.5 \%)$ & 0.56 & \\
\hline Abdominal pain & $17(34.7 \%)$ & $7(17.5 \%)$ & 0.07 & \\
\hline Vomiting & $9(18.4 \%)$ & $3(7.5 \%)$ & 0.21 & \\
\hline Diarrhoea & $10(20.4 \%)$ & $3(7.5 \%)$ & 0.13 & \\
\hline Anorexia & $7(14 \%)$ & $4(10 \%)$ & 0.75 & \\
\hline COVID respiratory disease & $42(80.8 \%)$ & $42(85.7 \%)$ & 0.51 & \\
\hline Hospital regimen & & & 0.84 & \\
\hline Intensive care unit, n (\%) & $18(34.6 \%)$ & $16(32.7 \%)$ & & \\
\hline Subintensive care, n (\%) & $34(65.4 \%)$ & $33(67.3 \%)$ & & \\
\hline \multicolumn{5}{|l|}{ Treatments during admission } \\
\hline Antibiotics/antimicotic & $43 / 50(86 \%)$ & $42 / 46(91.3 \%)$ & 0.42 & \\
\hline Antiviral & $27 / 48(56.2 \%)$ & $15 / 44(34.1 \%)$ & 0.03 & 2.49 (1.07 to 5.79$)$ \\
\hline Hydroxychloroquine & $21 / 49(42.9 \%)$ & $19 / 44(43.2 \%)$ & 0.98 & \\
\hline Biologic therapy & $11 / 48(22.9 \%)$ & $3 / 43(7 \%)$ & 0.04 & 3.96 (1.03 to 15.33$)$ \\
\hline Anticoagulation & $26 / 42(61.9 \%)$ & $21 / 37(56.8 \%)$ & 0.64 & \\
\hline Steroids & $15 / 50(30 \%)$ & $13 / 45(28.9 \%)$ & 0.90 & \\
\hline
\end{tabular}

${ }^{*}$ Among patients with available information.

ASA, American Society of Anesthesiologists; CKD, Chronic Kidney Disease; COPD, Chronic obstructive pulmonary disease; NSAIDS, Nonsteroidal anti-inflammatory drug; UGI, Upper Gastrointestinal. 


\section{Association between GI findings and prognosis}

Twenty-three patients $(21.7 \%)$ deceased during admission after a median of 15 (6-22) days from endoscopy. Excluding patients with AoC findings for their background fragility (mortality rate $=38.5 \%$ ), mortality among patients with major findings versus those with 'negative' procedure was $23.5 \%$ vs $14.3 \%, \mathrm{p}=0.3$.

\section{DISCUSSION}

In this multicentre cross-sectional study including all consecutive patients with COVID-19 requiring endoscopy in 16 centres in high-incidence areas during the first pandemic phase, almost half were found with acute mucosal injuries. Many of these findings were found early during admission and did not seem to be related to prolonged hospitalisation. None of the hospitalisation-related or patient-related variables registered in our cohort were associated with this risk, as opposed to elevated D-dimer values and GI symptoms.

While initial reports on COVID-19 have understandably focused on respiratory illness, more than $10 \%{ }^{4-6}$ of patients have been observed to self-report at least one digestive symptom at admission, even without or before the onset of respiratory manifestations; ${ }^{6} 7$ SARS-CoV-2 can be detected in faecal samples of most patients with COVID-1911 1227 and even in stools of patients recovered from infection during convalescence. ${ }^{5}$ Despite this increasing awareness of GI involvement and the potential risk carried by GI endoscopy, ${ }^{28}$ evidence on endoscopic findings in patients with COVID-19 is extremely limited. ${ }^{17}$

In one series of only six cases of patients with COVID-19 with GI symptoms undergoing endoscopy, oesophageal bleeding with erosions and ulcers were described in one patient although SARS-CoV-2 RNA was detected in most patients either in the stomach, duodenum or rectum specimens. ${ }^{9} 29$

While drafting this manuscript, an Italian retrospective series of endoscopic evaluation on patients with COVID-19 has been published. ${ }^{30}$ The authors found a GI mucosal damage in $75 \%$ of patients, with a $20 \%$ prevalence of histologically confirmed ischaemic colitis. Those results are partially in line with those reported in our paper, which likely represents a less biased evaluation due to the prospective enrolment and the inclusion of patients from international centres in different high-prevalence geographical areas. Moreover, the larger sample size and the prospective enrolment have permitted the realtime assessment of several confounders and, therefore, the possibility of inferences on possible etiopathogenic mechanisms of GI injury.

In an Italian cohort of almost 5000 patients with COVID-19, 23 presented UGI bleeding $(0.47 \%)$, of whom half were investigated with UGI endoscopy, with peptic ulcer as the most prevalent finding. ${ }^{31}$

Expressed by intestinal epithelium, ACE-2 acts as a viral entry receptor and is furthermore involved in regulating intestinal inflammation and secretions, possibly explaining why GI symptoms are frequently described. ${ }^{6-1029}$ The distribution of the ACE-2 receptor, however, may also point toward indirect mechanisms of GI involvement. Indeed, ACE-2 receptor is expressed in endothelial cells ${ }^{32}$ that can be infected with consequent microvascular damage leading to vasoconstriction and propagation of microthrombi leading to ischaemia. ${ }^{33}$ It has indeed been shown that severe endothelial injury, thrombotic microangiopathy and angiogenesis peculiarly distinguishes COVID-19 lung pathology from that of equally severe influenza virus infections. ${ }^{19}$ Moreover, viral invasion incites an anti-viral inflammatory response which eventually results in clinical deterioration (through the so-called 'cytokine storm'). This extreme inflammatory response, the ischaemic damage and the imbalance towards a procoagulant environment can potentially affect almost every organ and may explain the broad spectrum of clinical features of this disease. ${ }^{20}{ }^{33}$ Furthermore it has been clearly demonstrated that increased D-dimer levels, reduced platelet count and prolonged prothrombin time, indicating a consumption coagulopathy, are associated with increased mortality among patients with COVID-19. This likely also explains why anticoagulant regimens seem to be protective ${ }^{24} 34$ although the reasons for these associations are yet to be fully clarified. Besides direct viral invasion and the cytokine storm, liver dysfunction or virus-induced antiphospholipid antibodies are possible additional pathogenic events. ${ }^{34}$ COVID-19-associated damage in the GI-tract might either be specific or secondary to microvascular impairment affecting intestinal perfusion. Notably, in patients with COVID-19, active diarrhoea has been associated with intestinal inflammation (through faecal calprotectin) and systemic interleukin-6 response, but not with RNA detection in stool. ${ }^{35}$ Endothelitis of submucosal vessels in patients with small instestine ischaemia has been reported at histopathological analysis after bowel resection. $^{32}$

We therefore hypothesised that endoscopic abnormalities in the upper and lower GI tract might be observed in a sizeable part of patients with COVID-19 possibly through ischaemic damage.

In this highly selected cohort of patients with COVID-19 requiring endoscopy during a restricted endoscopic practice, we found that almost half of patients had a major abnormality. This category included severe mucosal damage, namely ulcerative or bleeding conditions not attributed to pre-existing conditions. Among UGI endoscopies, $10 \%$ reported a petechial/haemorrhagic gastropathy, with almost $30 \%$ showing gastroduodenal ulcers (63.6\% actively or recently bleeding) and $15 \%$ with diffuse erosive damage. Among LGI endoscopies, one-third was found with an ischaemic-like colopathy and $10 \%$ with acute bleeding of undetected origin. This high prevalence of mucosal injury must be read in light of the likely selection of the most severe patients, as only a small fraction $(<1 \%)$ of admitted patients with SARS-CoV-2 have been endoscopically investigated in an era in which deferral of elective procedures has led to 


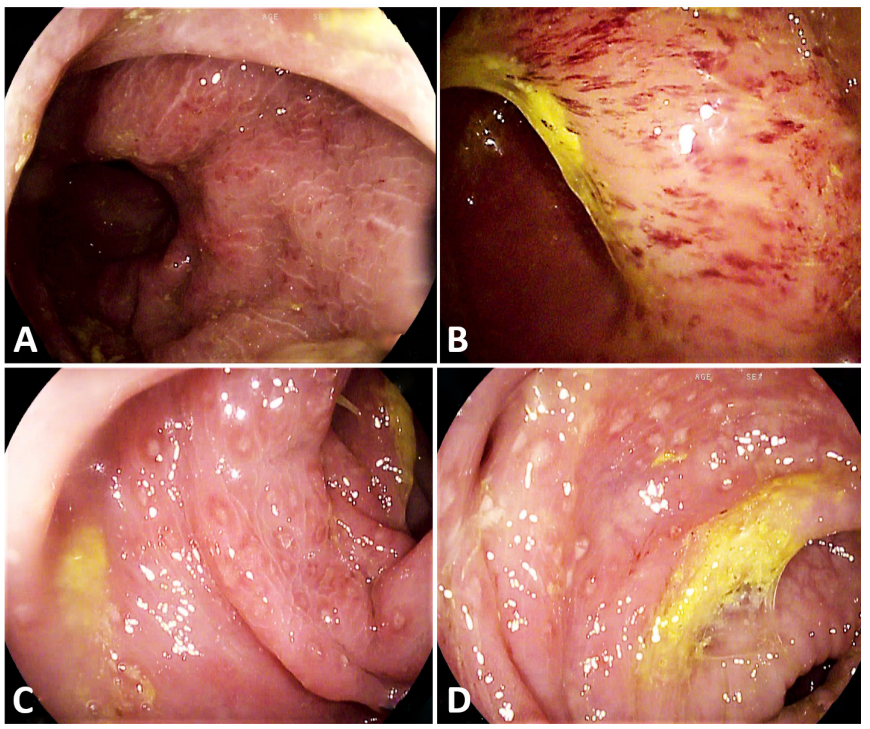

Figure 7 Colonoscopy of a young patient performed for persisting diarrhoea 2 months after being discharged from a COVID-19 ward. In the rectum, a fragile and dystrophic mucosa with diffuse petechiae was seen (A, B). The mucosa of the distal sigmoid colon appeared severely oedematous, congested, with diffuse aphthous erosions (C, D). Histology of the whole colon showed intense lymphocytic and granulomatous infiltration.

extreme contraction of endoscopy volumes. ${ }^{13-15}$ Of note, severe oedema, congestion, petechiae and aphthous erosions were also found in one patient recovered from COVID-19 infection, undergoing elective colonoscopy due to persistent diarrhoea (see figure 7).

As for the lower tract, agreement for the interpretation of abnormalities as 'ischaemic-like' is usually higher among expert endoscopists due to typical findings and distribution. ${ }^{26}$ Moreover, this hypothesis was histologically confirmed in $80 \%$ of suspected cases in which biopsies were taken.

Conversely, despite UGI damage reported in our series are compatible with reports of GI mucosal injuries among patients with critical illness ${ }^{36}$ for which hypoperfusion is the major underlying mechanism, ${ }^{37}$ unequivocal interpretation into a pathognomonic condition is less widely accepted and we preferred a descriptive report. As some of these endoscopic abnormalities might be theoretically due to drug-induced damage or to intensive care admission per se, ${ }^{1737}$ we included these variables in the multivariate logistic regression analysis but could not demonstrate any role for pre-existing frailty, in-hospital treatments or intensity of care. On the other hand, the presence of GI symptoms, which may represent a consequence, instead of a risk factor, and increased D-dimer values were associated with an increased risk of acute major findings. D-dimers are a product of fibrin degradation, and a sensitive biomarker of activation of coagulative cascades and elevated values have been ascertained as an independent biomarker for poor prognosis (increased severity and mortality) in patients with COVID-19. ${ }^{38}$
The present findings are also in line with preliminary reports of radiological findings in patients with COVID$19 .^{39}$ In one series of hospitalised patients undergoing cross-sectional imaging 39 of patients undergoing CT-scan were found with bowel wall thickening, and $20 \%$ with pneumatosis or portal venous gas, indicating a mesenteric ischaemia, which was also detectable macroscopically and/or histologically in patients needing surgery.

This study has several limitations.

First of all, as already discussed, the study period included the first pandemic outbreak, during which access to endoscopy was severely restricted. ${ }^{13-15}$ Indeed, patient with COVID-19 included in this study were $<1 \%$ of admitted patients with SARS-CoV-2 in the included institutions, therefore representing a specific subset of more 'severe' patients in which endoscopy was deemed urgent or not deferrable. All the findings and inferences must therefore be read in light of this significant selection bias.

Second, due to many procedures being urgent or performed to exclude or treat a GI bleeding, histological samples were taken in only a minority of cases.

Third, COVID-19 severity is not optimally evaluated in this study. An increasing body of evidence has nowadays shown that multiple demographic (age, sex, smoking status, comorbidities), clinical (blood pressure, hypoxaemia, tachycardia, etc) and biochemical (procalcitonin, C-reactive protein, aspartate aminotransferase, lactate, creatinine, white blood cells count, etc) variables can help in predict the disease course and prognosis of COVID-19. ${ }^{40-43}$ Unfortunately, this study was designed as a prospective collection at the very beginning of the first pandemic wave, and therefore only few potentially relevant factors could be registered as indicators of a possible diseases severity. ${ }^{37}$ Furthermore, the discriminating value of D-dimers in our series is suboptimal (AUC=0.619) and the obtained threshold is inadequate to be used as a single diagnostic test.

Fourth, most of patients found with an ischaemic-like injury, could not undergo a comprehensive and thorough evaluation of eventual concomitant risk factors (eg, through CT angiography), this questioning the causal role of SARS-CoV-2 infection in these events.

Fifth, despite this cohort present the results of a multicentre collaboration, $59 \%$ of included examinations came from one single country (Italy) and therefore might not be reproducible in a different case mix.

For all the above mentioned reasons, our results must be read as descriptive and exploratory, without having the power to confirm an association between SARS-CoV-2 infection and ischaemic GI tract injury. However, our report of ischaemic phenomena even in patients without previous comorbidities, the impossibility to demonstrate a role for pre-existing frailty or intensity of care in these events and the fact that endothelial injury has been described as a peculiar characteristic of SARS-CoV-2 infection, ${ }^{19}$ at least highlights the possibility that in a subset of 
patients with COVID-19, gastrointestinal ischaemic injury can happen in the absence of common risk factors.

Finally, since the study was conceived as an endoscopic registry and lacks long-term follow-up, it is underpowered to prove a prognostic relevance of these findings. However, our preliminary data on D-dimers suggest the hypothesis that gastrointestinal damage might reflect a systemic pro-coagulative status, which is associated with a worse prognosis. ${ }^{24}$ Moreover, clinical GI involvement in patients with COVID-19 has already been reported to carry a prognostic relevance, with patients with GI symptoms experiencing more severe clinical outbreaks and higher need for intensive treatments. ${ }^{44}$ Considering the high rate of symptomatic patients in our selected cohort $(40 \% \text { vs an average reported prevalence of } 15 \%)^{4}$ and symptoms being an independent predictor of acute findings in our regression analysis, it is tempting to speculate that an endoscopically detected damage might reflect a more aggressive disease course, associated with a worse prognosis.

Despite all these limitations, to the best of our knowledge, this is the first international multicentre experience describing endoscopy findings in a relatively large cohort of patients with COVID-19, increasing knowledge on sites and patterns of GI tract involvement. The prospective nature of the registry reassures about the inclusion of all consecutive procedures relevant for the study aim.

In parallel, the study speculates the hypothesis of a systemic inflammatory and pro-coagulative environment as the primer for gastrointestinal injury, deserving further exploration through less selected cohorts, autoptic series and more thorough clinical and translational investigation, since an eventual ischaemic aetiopathogenesis of COVID-19 gastrointestinal involvement potentially carries relevant therapeutic and preventive implications.

\section{Author affiliations}

${ }^{1}$ Pancreatobiliary Endoscopy and Endosonography Division, IRCCS San Raffaele Scientific Institute, Vita-Salute San Raffaele University, Milan, Italy

${ }^{2}$ Digestive Endoscopy Unit, ASST Papa Giovanni XXIII, Bergamo, Italy

${ }^{3}$ Gastroenterology and Gastrointestinal Endoscopy Division, IRCCS San Raffaele Scientific Institute, Vita-Salute San Raffaele University, Milan, Italy

${ }^{4}$ Department of Medical and Surgical Sciences, University of Bologna and Sant'Orsola Malpighi Hospital, Bologna, Italy

${ }^{5} \mathrm{GI}$ Endoscopy Service, Hospital Casa de Saude de Santos, Santos, Brazil

${ }^{6}$ Digestive Endoscopy Unit, Fondazione Policlinico Universitario Agostino Gemelli IRCCS, Rome, Italy

${ }^{7}$ Center for Endoscopic Research Therapeutics and Training (CERTT), Università Cattolica del Sacro Cuore, Rome, Italy

${ }^{8}$ Department of Gastroenterology and Hepatology, University Hospitals Leuven, Leuven, Belgium

${ }^{9}$ Robert Wood Johnson Medical School Rutgers University, New Brunswick, New Jersey, USA

${ }^{10}$ Section of Digestive Diseases, Department of Internal Medicine, Yale University School of Medicine, New Haven, Connecticut, USA

${ }^{11}$ Digestive Endoscopy Unit, Sant'Andrea Hospital, Sapienza University, Rome, Italy

${ }^{12}$ Department of Internal Medicine, San Matteo Hospital Foundation, University of Pavia, Pavia, Italy

${ }^{13}$ Gastroenterology Unit, Department of Surgery, Oncology and Gastroenterology, University of Padua, Padua, Italy

${ }^{14}$ Department of Gastroenterology, Newcastle upon Tyne hospitals NHS Trust, Newcastle upon Tyne, UK
${ }^{15}$ Department of Gastroenterology and Hepatology, University Hospital of Santiago de Compostela. Health Research Institute of Santiago de Compostela (IDIS),

Santiago de Compostela, Spain

${ }^{16}$ Imelda GI Clinical Research Center, Imeldaziekenhuis, Bonheiden, Belgium

${ }^{17}$ National and Kapodistrian University of Athens, "Attikon" University General Hospital, Athens, Greece

${ }^{18}$ Ospedale Sandro Pertini, Rome, Italy

${ }^{19}$ Department of Hematology and Stem Cell Transplantation, IRCCS San Raffaele

Scientific Institute, Vita-Salute San Raffaele University, Milan, Italy

${ }^{20}$ Division of Immunology, Transplantation and Infectious Diseases, IRCCS San

Raffaele Scientific Institute, Vita-Salute San Raffaele University, Milan, Italy

${ }^{21}$ Department of Chronic Disease, Metabolism and Ageing (CHROMETA), KU Leuven, Leuven, Belgium

Twitter Pierluigi Fracasso @pigifracasso

Acknowledgements The authors thank Dr Livia Archibugi (San Raffaele Scientific Institute, Milan, Italy) and Dr Antonella Toma (Ospedale Sandro Pertini, Rome, Italy) for their contribution to the iconographic material included in this manuscript.

Contributors PGA and GC were involved in study concept and design. SG, JF, ELDAA, ADS, MK, PR, CB, LF, LR, ASL, IB, MB, AT, GKKN, SA, AM, FZ, KWO, DdIIG, LP, ISP, PF, CT, EV, LHE, MER, SWVdM, HS, AS, JWY, ED, RAS, FA, FB, SP, MCP and $P R Q$ were involved in acquisition of data. GV and GC were involved in statistical analysis. GV, GC and PGA were involved in analysis and interpretation of data and in drafting of the manuscript. MB, SWvdM, KWO, ES, ADS, JF, MK, GC, JIG, EDG, ELDAA, FB, PAT, SG, FC and PGA were involved in critical revision of the manuscript for important intellectual content. All authors revised the manuscript, approved the final version to be published and agree to be accountable for accuracy and integrity of any part of the work. Guarantor of the article: PGA.

Funding The authors have not declared a specific grant for this research from any funding agency in the public, commercial or not-for-profit sectors.

Competing interests GV received travel grants from Mylan and Alfasigma. GC is a consultant for Mylan. IB is consultant for Apollo Endosurgery, Cook Medical and Boston Scientific; board member for Endo Tools; research grant recipient from Apollo Endosurgery; had food and beverage compensation from Apollo Endosurgery, Cook Medical, Boston Scientific and Endo Tools. LR is a consultant for Cancer Prevention Pharmaceuticals; has received research grants from SLA Pharma AG and Takeda and receives funds from the Italian Association for Cancer Research (IG21723). MB received travel grants from Takeda, Taewoong Medical and Prion Medical. KWO has received lecture fees from Olympus, Medtronic and Mylan. He has received a research grant from Medtronic. LP received advisory board fees from Janssen and Takeda; presentation fees from AbbVie and Ferring; and personal fees from AbbVie, Ferring, Norgine and Takeda. SWVdM holds the Cook chair in interventional endoscopy and holds consultancy agreements with Boston Scientific, Cook, Pentax and Olympus. ES has received lecture or consultancy fees from Medtronic, Reckitt Benckiser, Takeda, Merck \& Co, Bristol Myers Squibb, AbbVie, Amgen, Novartis, Fresenius Kabi, Sandoz, Sofar, Malesci, Janssen, Grifols, Aurora Pharma, Innovamedica, Johnson \& Johnson, SILA, Unifarco, Alfasigma, Shire, EG Stada Group. MK has done consulting work for Boston Scientific, Interscope Med and AbbVie. He has received research grants from Boston Scientific, Emcision, Conmed, Pinnacle, Cook, Gore, Merit and Olympus. PR is supported by Clinical Mandate from Belgian Foundation against Cancer (Stichting tegen Kanker) and receives speaking and consultancy fees from MSD Belgium. GC is consultant for and had food and beverage compensation from Cook Medical, Boston Scientific and Olympus.

Patient consent for publication Not required.

Ethics approval IRCCS San Raffaele Institute promoted the study and obtained local Institutional Review Board (IRB) approval under the code COVID-BioB (n.34/int/2020) as part of a general protocol registered in ClinicalTrial.gov. Each participating centre obtained approval in keeping with local IRB policy.

Provenance and peer review Not commissioned; externally peer reviewed.

Data availability statement All data relevant to the study are included in the article or uploaded as supplementary information.

Supplemental material This content has been supplied by the author(s). It has not been vetted by BMJ Publishing Group Limited (BMJ) and may not have been peer-reviewed. Any opinions or recommendations discussed are solely those of the author(s) and are not endorsed by BMJ. BMJ disclaims all liability and responsibility arising from any reliance placed on the content. Where the content includes any translated material, BMJ does not warrant the accuracy and reliability 
of the translations (including but not limited to local regulations, clinical guidelines, terminology, drug names and drug dosages), and is not responsible for any error and/or omissions arising from translation and adaptation or otherwise.

Open access This is an open access article distributed in accordance with the Creative Commons Attribution Non Commercial (CC BY-NC 4.0) license, which permits others to distribute, remix, adapt, build upon this work non-commercially, and license their derivative works on different terms, provided the original work is properly cited, appropriate credit is given, any changes made indicated, and the use is non-commercial. See: http://creativecommons.org/licenses/by-nc/4.0/.

ORCID iD

Giuseppe Vanella http://orcid.org/0000-0001-7280-1761

\section{REFERENCES}

1 Ciceri F, Beretta L, Scandroglio AM, et al. Microvascular COVID-19 lung vessels obstructive thromboinflammatory syndrome (MicroCLOTS): an atypical acute respiratory distress syndrome working hypothesis. Crit Care Resusc 2020;22:95-97.

2 Xie Y, Bowe B, Maddukuri G, et al. Comparative evaluation of clinical manifestations and risk of death in patients admitted to hospital with covid-19 and seasonal influenza: cohort study. BMJ 2020;371:m4677.

3 Puelles VG, Lütgehetmann M, Lindenmeyer MT, et al. Multiorgan and renal tropism of SARS-CoV-2. N Engl J Med 2020;383:590-2.

4 Mao R, Qiu Y, He J-S, et al. Manifestations and prognosis of gastrointestinal and liver involvement in patients with COVID-19: a systematic review and meta-analysis. Lancet Gastroenterol Hepatol 2020;5:667-78.

5 Cheung KS, Hung IFN, Chan PPY, et al. Gastrointestinal manifestations of SARS-CoV-2 infection and virus load in fecal samples from a Hong Kong cohort: systematic review and metaanalysis. Gastroenterology 2020;159:81-95.

6 Sultan S, Altayar O, Siddique SM, et al. AGA Institute rapid review of the gastrointestinal and liver manifestations of COVID-19, meta-analysis of international data, and recommendations for the consultative management of patients with COVID-19. Gastroenterology 2020;159:e27:320-34.

7 Pan L, Mu M, Yang P, et al. Clinical characteristics of COVID-19 patients with digestive symptoms in Hubei, China: a descriptive, cross-sectional, multicenter study. Am J Gastroenterol 2020;115:766-73

8 Zhang H, Kang Z, Gong $\mathrm{H}$, et al. Digestive system is a potential route of COVID-19: an analysis of single-cell coexpression pattern of key proteins in viral entry process. Gut 2020;69:1010-8.

9 Xiao F, Tang M, Zheng X, et al. Evidence for gastrointestinal infection of SARS-CoV-2. Gastroenterology 2020;158:1831-3.

10 ACE2 protein expression summary - The Human Protein Atlas. Available: https://www.proteinatlas.org/ENSG00000130234-ACE2 [Accessed 20 Dec 2020].

11 Gu J, Han B, Wang J. COVID-19: gastrointestinal manifestations and potential fecal-oral transmission. Gastroenterology 2020;158:1518-9.

12 Wong MC, Huang J, Lai C, et al. Detection of SARS-CoV-2 RNA in fecal specimens of patients with confirmed COVID-19: a metaanalysis. J Infect 2020;81:e31-8.

13 ASGE. Gastroenterology professional Society guidance on endoscopic procedures during the COVID-19 pandemic. Available: https://www.asge.org/home/resources/key-resources/covid19-asge-updates-for-members/gastroenterology-professionalsociety-guidance-on-endoscopic-procedures-during-the-covid-19pandemic [Accessed 20 Dec 2020]

14 Gralnek IM, Hassan C, Beilenhoff U, et al. ESGE and ESGENA position statement on gastrointestinal endoscopy and the COVID-19 pandemic. Endoscopy 2020;52:483-90.

15 Chiu PWY, Ng SC, Inoue H, et al. Practice of endoscopy during COVID-19 pandemic: position statements of the Asian Pacific Society for digestive endoscopy (APSDE-COVID statements). Gut 2020;69:991-6.

16 The British Society of Gastroenterology. Endoscopy activity and COVID-19: Bsg and JAG guidance - update 22.03.20. Available: https://www.bsg.org.uk/covid-19-advice/endoscopy-activity-andcovid-19-bsg-and-jag-guidance/ [Accessed 3 Apr 2020].

17 Lin L, Jiang X, Zhang Z, et al. Gastrointestinal symptoms of 95 cases with SARS-CoV-2 infection. Gut 2020;69:997-1001.

$18 \mathrm{Ng} \mathrm{SC}$, Tilg H. COVID-19 and the gastrointestinal tract: more than meets the eye. Gut 2020;69:973-4.
19 Ackermann M, Verleden SE, Kuehnel M, et al. Pulmonary vascular Endothelialitis, thrombosis, and angiogenesis in Covid-19. N Engl J Med 2020;383:120-8.

20 Cardinale V, Capurso G, laniro G, et al. Intestinal permeability changes with bacterial translocation as key events modulating systemic host immune response to SARS-CoV-2: a working hypothesis. Dig Liver Dis 2020;52:1383-9.

21 Laboratory testing for 2019 novel coronavirus (2019-nCoV) in suspected human cases. Available: https://www.who.int/ publications-detail/laboratory-testing-for-2019-novel-coronavirus-insuspected-human-cases-20200117 [Accessed 26 Apr 2020].

22 ASA Physical Status Classification System. American Society of Anesthesiologists (ASA). Available: https://www.asahq.org/ standards-and-guidelines/asa-physical-status-classification-system [Accessed 9 May 2020].

23 Jean S-S, Lee P-I, Hsueh P-R. Treatment options for COVID-19: the reality and challenges. J Microbiol Immunol Infect 2020;53:436-43.

24 Giannis D, Ziogas IA, Gianni P. Coagulation disorders in coronavirus infected patients: COVID-19, SARS-CoV-1, MERS-CoV and lessons from the past. J Clin Virol 2020;127:104362.

25 Minimal Standard Terminology. World endoscopy organization (WEO). Available: http://www.worldendo.org/resources/minimalstandard-terminology-mst/ [Accessed 3 Apr 2020].

26 Doulberis M, Panagopoulos P, Scherz S, et al. Update on ischemic colitis: from etiopathology to treatment including patients of intensive care unit. Scand J Gastroenterol 2016;51:893-902.

27 Pan Y, Zhang D, Yang P, et al. Viral load of SARS-CoV-2 in clinical samples. Lancet Infect Dis 2020;20:411-2.

28 European Society of Gastrointestinal Endoscopy (ESGE). ESGE and ESGENA position statement on gastrointestinal endoscopy and the COVID-19 pandemic. Available: https://www.esge.com/esge-andesgena-position-statement-on-gastrointestinal-endoscopy-and-thecovid-19-pandemic/ [Accessed 3 Apr 2020].

29 Liang W, Feng Z, Rao S, et al. Diarrhoea may be underestimated: a missing link in 2019 novel coronavirus. Gut 2020;69:1141-3.

30 Massironi S, Viganò C, Dioscoridi L, et al. Endoscopic findings in patients infected with 2019 novel coronavirus in Lombardy, Italy. Clin Gastroenterol Hepatol 2020;18:2375-7.

31 Mauro A, De Grazia F, Lenti MV, et al. Upper gastrointestinal bleeding in COVID-19 inpatients: incidence and management in a multicenter experience from northern Italy. Clin Res Hepatol Gastroenterol 2020:101521

32 Varga Z, Flammer AJ, Steiger P, et al. Endothelial cell infection and endotheliitis in COVID-19. The Lancet 2020;395:1417-8.

33 Wadman M, Couzin-Frankel J, Kaiser J. How does coronavirus kill? clinicians trace a ferocious rampage through the body, from brain to toes Science 2020.

34 Bikdeli B, Madhavan MV, Jimenez D, et al. COVID-19 and thrombotic or thromboembolic disease: implications for prevention, antithrombotic therapy, and follow-up: JACC state-of-the-art review. J Am Coll Cardiol 2020;75:2950-73.

35 Effenberger M, Grabherr F, Mayr L, et al. Faecal calprotectin indicates intestinal inflammation in COVID-19. Gut 2020;69:1543-4

36 Ovenden C, Plummer MP, Selvanderan S, et al. Occult upper gastrointestinal mucosal abnormalities in critically ill patients. Acta Anaesthesiol Scand 2017;61:216-23.

37 Bardou M, Quenot J-P, Barkun A. Stress-Related mucosal disease in the critically ill patient. Nat Rev Gastroenterol Hepatol 2015:12:98-107.

38 Zhang L, Yan X, Fan Q. D-dimer levels on admission to predict in-hospital mortality in patients with Covid-19. J Thromb Haemost. Published online 2020.

39 Bhayana R, Som A, Li MD, et al. Abdominal imaging findings in COVID-19: preliminary observations. Radiology 2020;297:E207-15.

40 Altschul DJ, Unda SR, Benton J, et al. A novel severity score to predict inpatient mortality in COVID-19 patients. Sci Rep 2020;10:16726.

41 Zeng F, Huang Y, Guo Y, et al. Association of inflammatory markers with the severity of COVID-19: a meta-analysis. Int $J$ Infect Dis 2020:96:467-74.

42 Tjendra Y, Al Mana AF, Espejo AP, et al. Predicting disease severity and outcome in COVID-19 patients: a review of multiple biomarkers. Arch Pathol Lab Med 2020;144:1465-74.

43 Izcovich A, Ragusa MA, Tortosa F, et al. Prognostic factors for severity and mortality in patients infected with COVID-19: a systematic review. PLoS One 2020;15:e0241955

44 D'Amico F, Baumgart DC, Danese S. Diarrhea during COVID-19 infection: pathogenesis, epidemiology, prevention and management. Clin Gastroenterol Hepatol 2020;18. 